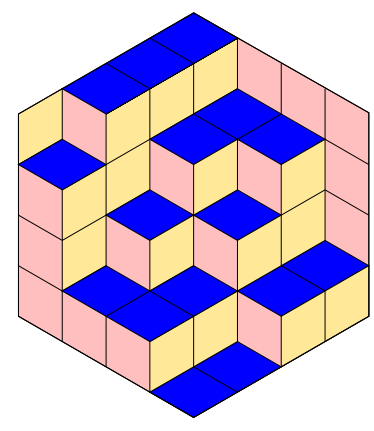

ALGEBRAIC COMBINATORICS

Sami Assaf \& Anne Schilling

A Demazure crystal construction for Schubert polynomials

Volume 1, issue 2 (2018), p. 225-247.

<http://alco.centre-mersenne.org/item/ALCO_2018__1_2_225_0>

(C) The journal and the authors, 2018.

Some rights reserved.

(c) BY This article is licensed under the

Creative Commons ATtribution 4.0 InTERnational License.

http://creativecommons.org/licenses/by/4.0/

Access to articles published by the journal Algebraic Combinatorics on the website http://alco.centre-mersenne.org/ implies agreement with the Terms of Use (http://alco.centre-mersenne.org/legal/).

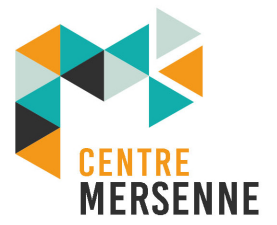

Algebraic Combinatorics is member of the Centre Mersenne for Open Scientific Publishing www.centre-mersenne.org 


\title{
A Demazure crystal construction for Schubert polynomials
}

\author{
Sami Assaf \& Anne Schilling
}

\begin{abstract}
Stanley symmetric functions are the stable limits of Schubert polynomials. In this paper, we show that, conversely, Schubert polynomials are Demazure truncations of Stanley symmetric functions. This parallels the relationship between Schur functions and Demazure characters for the general linear group. We establish this connection by imposing a Demazure crystal structure on key tableaux, recently introduced by the first author in connection with Demazure characters and Schubert polynomials, and linking this to the type A crystal structure on reduced word factorizations, recently introduced by Morse and the second author in connection with Stanley symmetric functions.
\end{abstract}

\section{INTRODUCTION}

Schubert polynomials $\mathfrak{S}_{w}$ were first introduced by Bernstein et al. [6] as certain polynomial representatives of cohomology classes of Schubert cycles $X_{w}$ in flag varieties. They were extensively studied by Lascoux and Schützenberger [15] using an explicit definition in terms of difference operators $\partial_{w}$. Subsequently, a combinatorial expression for Schubert polynomials as the generating polynomial for compatible sequences for reduced expressions of a permutation $w$ was discovered by Billey, Jockusch, and Stanley [7]. In the special case of the Grassmannian subvariety, Schubert polynomials are Schur polynomials, which also arise as the irreducible characters for the general linear group.

The Stanley symmetric functions $F_{w}$ were introduced by Stanley [27] in the pursuit of enumerations of the reduced expressions of permutations, in particular of the long permutation $w_{0}$. They are defined combinatorially as the generating functions of reduced factorizations of permutations. Stanley symmetric functions are the stable limit of Schubert polynomials [20,21], precisely

$$
F_{w}\left(x_{1}, x_{2}, \ldots\right)=\lim _{m \rightarrow \infty} \mathfrak{S}_{1^{m} \times w}\left(x_{1}, x_{2}, \ldots, x_{n+m}\right) .
$$

Edelman and Greene [10] showed that the coefficients of the Schur expansion of Stanley symmetric functions are nonnegative integer coefficients.

Demazure modules for the general linear group [9] are closely related to Schubert classes for the cohomology of the flag manifold. In certain cases these modules are irreducible polynomial representations, and so the Demazure characters also contain the Schur polynomials as a special case. Lascoux and Schützenberger [16] stated that

Manuscript received 11th August 2017, revised 21st November 2017, accepted 27th December 2017. KEYwORDS. Schubert polynomials, Demazure characters, Stanley symmetric functions, crystal bases. ACKNOWLEDGEMENTS. AS was partially supported by NSF grant DMS-1500050. 
Schubert polynomials are nonnegative sums of Demazure characters. This was proven by Reiner and Shimozono [25] using the right keys associated to Edelman-Greene insertion. Using a key tableaux interpretation for Demazure characters [3], Assaf [2] showed that the Edelman and Greene algorithm giving the Schur expansion of a Stanley symmetric function can be modified to a weak Edelman-Greene algorithm which gives the Demazure expansion of a Schubert polynomial.

In this paper, we deepen this connection and provide a converse to (1.1) by showing that Schubert polynomials are Demazure truncations of Stanley symmetric functions. Specifically, we show in Theorem 5.11 that the combinatorial objects underlying the Schubert polynomials, namely the compatible sequences, exhibit a Demazure crystal truncation of the full Stanley crystal of Morse and Schilling [24]. We prove this using Theorem 3.14, in which we give an explicit Demazure crystal structure on semi-standard key tableaux, which coincide with semi-skyline augmented fillings of Mason [22]. This, together with Theorem 5.10, in which we show that the crystal operators on reduced factorizations intertwine with (weak) Edelman-Greene insertion, proves our main result.

Lenart [18] defined crystal operators on RC graphs [5], which are closely related to compatible sequences, though it was not observed there that this structure is a Demazure crystal. Earlier, Reiner and Shimozono [26] defined $r$-pairings on factorized row-frank words that can now be interpreted as crystal operators, but again, this was not observed nor was it noted that this structure is a Demazure crystal structure. One could complete either of these perspectives to prove our main result, though we prefer the key tableaux approach given its simplicity, the natural crystal operators on these objects, and the connection with Edelman-Greene insertion.

This paper is structured as follows. In Section 2, we review the crystal structure on semi-standard Young tableaux and define Demazure crystals. In Section 3, we introduce new crystal operators on key tableaux and prove that this amounts to a Demazure crystal (Theorem 3.14). Section 4 is reserved for the review of Stanley symmetric functions, Edelman-Greene insertion and the crystal structure on reduced factorization, which underly the Stanley symmetric functions. Section 5 contains our main result (Theorem 5.11), namely a Demazure crystal structure on reduced factorizations with cutoff, which are equivalent to compatible sequences. This gives a Demazure crystal structure for Schubert polynomials and shows that Schubert polynomials are a Demazure truncation of Stanley symmetric functions.

\section{Crystal structure on tableaux}

We begin in Section 2.1 by reviewing the basics of Schur polynomials via the combinatorics of Young tableaux. In Section 2.2, we review the type $A$ crystal structure on semi-standard Young tableaux, and conclude in Section 2.3 with the definition of Demazure crystals.

2.1. Combinatorics of Schur polynomials. Given a partition $\lambda$, the Young $d i$ agram of shape $\lambda$ is the array of left-justified cells with $\lambda_{i}$ boxes in row $i$. Here we use French notation, where the rows weakly decrease in size from bottom to top in the Young diagram. A Young tableau is a filling of the cells of a Young diagram from some totally ordered alphabet (for example the set of positive integers) such that rows and columns weakly increase. A semi-standard Young tableau is a Young tableau with distinct column entries. Figure 1 provides an example of semi-standard Young tableaux of a fixed shape. 


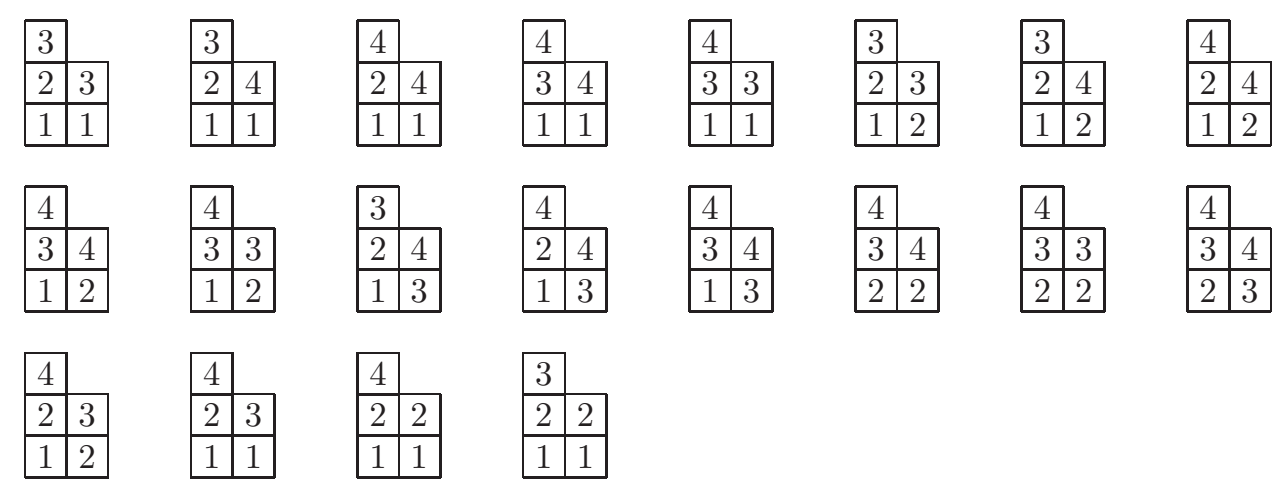

Figure 1. The semi-standard Young tableaux of shape $(2,2,1)$ over the alphabet $\{1,2,3,4\}$.

The weight of a semi-standard Young tableau $T$, denoted by $\operatorname{wt}(T)$, is the weak composition whose $i$ th part is the number of occurrences of $i$ in $T$. The shape $\lambda$ of $T$ is also denoted by $\operatorname{sh}(T)$.

Definition 2.1. The Schur polynomial in $n$ variables indexed by the partition $\lambda$ is

$$
s_{\lambda}(x)=s_{\lambda}\left(x_{1}, \ldots, x_{n}\right)=\sum_{T \in \operatorname{SSYT}_{n}(\lambda)} x_{1}^{\mathrm{wt}(T)_{1}} \cdots x_{n}^{\mathrm{wt}(T)_{n}},
$$

where $\operatorname{SSYT}_{n}(\lambda)$ is the set of semi-standard Young tableaux of shape $\lambda$ over the alphabet $\{1,2, \ldots, n\}$.

Schur polynomials arise as characters for irreducible highest weight modules for the general linear group with semi-standard Young tableaux giving a natural indexing set for the basis of the module.

2.2. Crystal operators on Semi-Standard Young tableaux. A crystal graph is a directed, colored graph with vertex set given by the crystal basis and directed edges given by deformations of the Chevalley generators. For the quantum group $U_{q}\left(\mathfrak{s l}_{n}\right)$, the crystal basis can be indexed by semi-standard Young tableaux over the alphabet $A=\{1,2, \ldots, n\}$ and there is an explicit combinatorial construction of the crystal graph on tableaux $[13,19]$. For an introduction to crystals from the quantum group perspective, see [11]. For a purely combinatorial introduction to crystals, see [8].

For a word $w$ of length $k$ with letters from the alphabet $A=\{1,2, \ldots, n\}$, an integer $0 \leqslant r \leqslant k$, and an integer $1 \leqslant i<n$, define

$$
M_{i}(w, r)=\operatorname{wt}\left(w_{1} w_{2} \cdots w_{r}\right)_{i}-\operatorname{wt}\left(w_{1} w_{2} \cdots w_{r}\right)_{i+1},
$$

where $\operatorname{wt}(w)$ is the weak composition whose $j$ th part is the number of $j$ 's in $w$. Set $M_{i}(w)=\max _{r}\left\{M_{i}(w, r)\right\}$. Observe that if $M_{i}(w)>0$ and $p$ is the leftmost occurrence of this maximum, then $w_{p}=i$, and if $q$ is the rightmost occurrence of this maximum, then either $q=k$ or $w_{q+1}=i+1$.

For a Young tableau $T$, the column reading word of $T$, denoted by $w(T)$, is the word obtained by reading the entries of $T$ down columns from left to right. For example, the column reading word of the leftmost Young tableau in the top row of Figure 1 is 32131.

Definition 2.2. Given an integer $1 \leqslant i<n$, define the lowering operator $f_{i}$ on semi-standard Young tableaux over the alphabet $A$ as follows: if $M_{i}(w(T)) \leqslant 0$, then 
$f_{i}(T)=0$; otherwise, let $p$ be the smallest index such that $M_{i}(w(T), p)=M_{i}(w(T))$, and $f_{i}(T)$ changes the entry in $T$ corresponding to $w(T)_{p}$ to $i+1$.

An example of the lowering operator $f_{2}$ is given in Figure 2. For this example, the column reading word is given below each semi-standard Young tableau with the largest index that attains $M_{2}(w(T))>0$ underlined and the corresponding entry in the tableau circled.

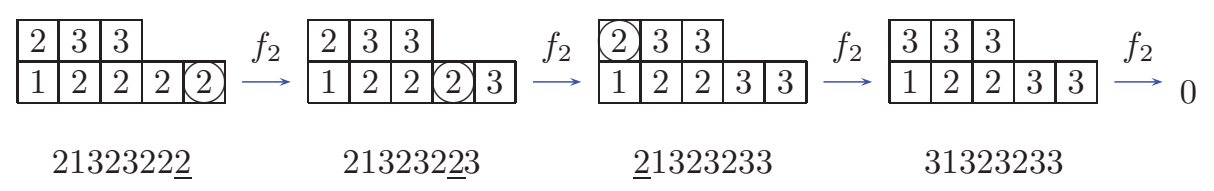

Figure 2. An example of the lowering operator $f_{2}$ on semi-standard Young tableaux.

DEFINITION 2.3. Given an integer $1 \leqslant i<n$, define the raising operator $e_{i}$ on semistandard Young tableaux over the alphabet $A$ as follows: let $q$ be the largest index such that $M_{i}(w(T), q)=M_{i}(w(T))$. If $q$ is the length of $w(T)$, then $e_{i}(T)=0$; otherwise, $e_{i}(T)$ changes the entry in $T$ corresponding to $w(T)_{q+1}$ to $i$.

For further examples of raising and lowering operators on semi-standard Young tableaux, see Figure 3. Note that we have drawn the crystal in Figure 3 with lowering operators pointing upward to facilitate the bijection with semi-standard key tableaux as explained in Section 3.

For a partition $\lambda$, we may define the highest weight crystal (of type $A_{n}$ ) of highest weight $\lambda$, denoted $B(\lambda)$, as the set $\operatorname{SSYT}_{n}(\lambda)$ together with the operators $f_{i}, e_{i}$ for $1 \leqslant i<n$ and the weight function wt. The character of a crystal is defined as

$$
\operatorname{ch} B(\lambda)=\sum_{b \in B(\lambda)} x_{1}^{\mathrm{wt}(b)_{1}} \cdots x_{n}^{\mathrm{wt}(b)_{n}},
$$

which in this case is precisely the Schur polynomial $s_{\lambda}\left(x_{1}, \ldots, x_{n}\right)$.

2.3. Demazure CRystals. Demazure characters first arose in connection with Schubert classes for the cohomology of the flag manifold in [9].

The divided difference operators $\partial_{i}$ for $1 \leqslant i<n$ act on polynomials by

$$
\partial_{i} f\left(x_{1}, \ldots, x_{n}\right)=\frac{f\left(x_{1}, \ldots, x_{i}, x_{i+1}, \ldots, x_{n}\right)-f\left(x_{1}, \ldots, x_{i+1}, x_{i}, \ldots, x_{n}\right)}{x_{i}-x_{i+1}} .
$$

For $w \in S_{n}$, we may define $\partial_{w}=\partial_{i_{1}} \partial_{i_{2}} \cdots \partial_{i_{k}}$ if $w=s_{i_{1}} s_{i_{2}} \cdots s_{i_{k}}$. Here $s_{i}(1 \leqslant i<n)$ is the simple transposition interchanging $i$ and $i+1$ and $k$ is the number of inversions (or length) of $w$. When $k$ is the length of $w$, the expression $s_{i_{1}} s_{i_{2}} \cdots s_{i_{k}}$ for $w$ is called a reduced expression. It can be shown that $\partial_{w}$ is independent of the choice of reduced expression.

There exist degree-preserving divided difference operators $\pi_{i}$ for $1 \leqslant i<n$, which act on polynomials by

$$
\pi_{i} f\left(x_{1}, \ldots, x_{n}\right)=\partial_{i}\left(x_{i} f\left(x_{1}, \ldots, x_{n}\right)\right) .
$$

As with $\partial_{i}$, we extend this definition to $w \in S_{n}$, by $\pi_{w}=\pi_{i_{1}} \pi_{i_{2}} \cdots \pi_{i_{k}}$ if $w=$ $s_{i_{1}} s_{i_{2}} \cdots s_{i_{k}}$ is a reduced expression, and $\pi_{w}$ is independent of the choice of reduced expression. 


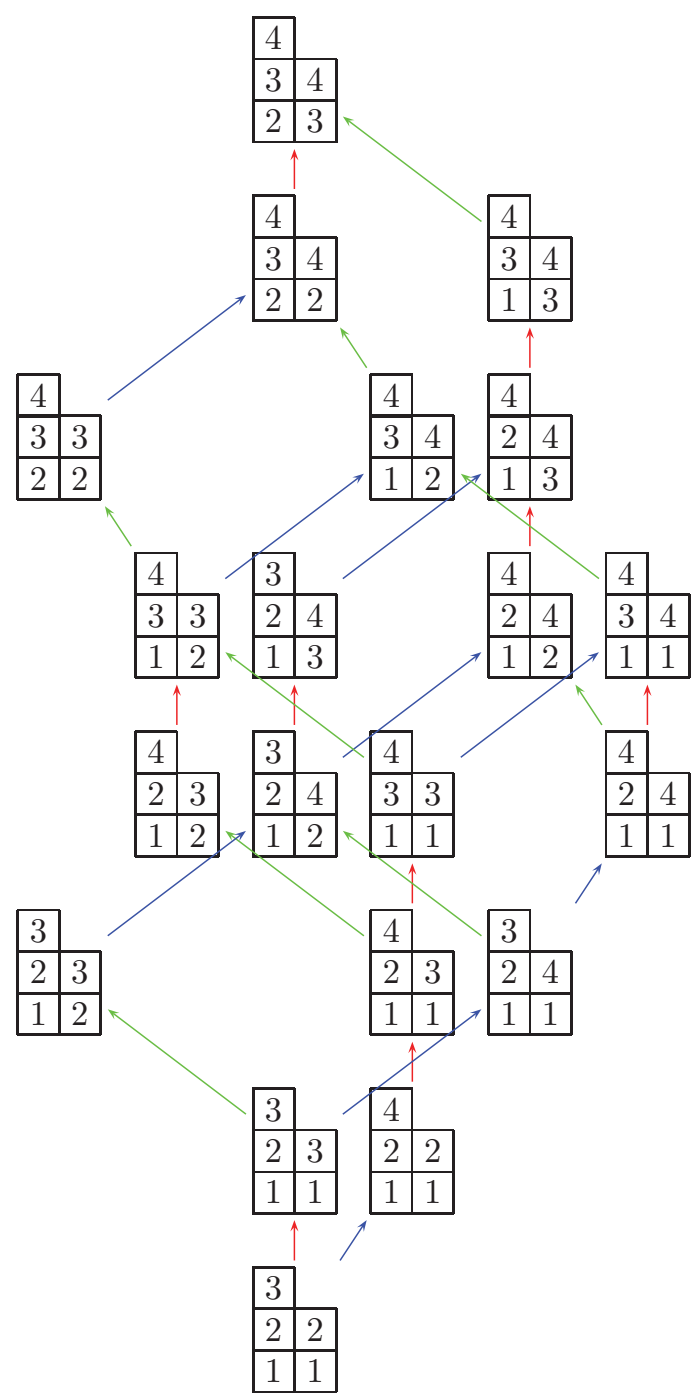

Figure 3 . The crystal $B(2,2,1)$, with edges $f_{1} \nwarrow, f_{2} \uparrow, f_{3} \nearrow$.

Definition 2.4. Given a weak composition a of length $n$, the Demazure character $\kappa_{a}$ is defined as

$$
\kappa_{a}(x)=\kappa_{a}\left(x_{1}, \ldots, x_{n}\right)=\pi_{w}\left(x_{1}^{\lambda_{1}} x_{2}^{\lambda_{2}} \cdots x_{n}^{\lambda_{n}}\right)
$$

where $\lambda$ is the partition rearrangement of $a$ and $w$ is the shortest permutation such that $\left(a_{w_{1}}, \ldots, a_{w_{n}}\right)=\lambda$. 
For example, we may compute the Demazure character $\kappa_{(0,2,1,2)}$ by taking $a=$ $(0,2,1,2), \lambda=(2,2,1,0)$ and $w=2431$, and so we have

$$
\begin{aligned}
& \kappa_{(0,2,1,2)} \\
& =\pi_{1} \pi_{3} \pi_{2} \pi_{3}\left(x_{1}^{2} x_{2}^{2} x_{3}\right) \\
& =\pi_{1} \pi_{3} \pi_{2}\left(x_{1}^{2} x_{2}^{2} x_{3}+x_{1}^{2} x_{2}^{2} x_{4}\right) \\
& =\pi_{1} \pi_{3}\left(x_{1}^{2} x_{2}^{2} x_{3}+x_{1}^{2} x_{2}^{2} x_{4}+x_{1}^{2} x_{2} x_{3}^{2}+x_{1}^{2} x_{2} x_{3} x_{4}+x_{1}^{2} x_{3}^{2} x_{4}\right) \\
& =\pi_{1}\left(x_{1}^{2} x_{2}^{2} x_{3}+x_{1}^{2} x_{2}^{2} x_{4}+x_{1}^{2} x_{2} x_{3}^{2}+2 x_{1}^{2} x_{2} x_{3} x_{4}+x_{1}^{2} x_{2} x_{4}^{2}+x_{1}^{2} x_{3}^{2} x_{4}+x_{1}^{2} x_{3} x_{4}^{2}\right) \\
& =x_{1}^{2} x_{2}^{2} x_{3}+x_{1}^{2} x_{2}^{2} x_{4}+x_{1}^{2} x_{2} x_{3}^{2}+2 x_{1}^{2} x_{2} x_{3} x_{4}+x_{1}^{2} x_{2} x_{4}^{2}+x_{1}^{2} x_{3}^{2} x_{4}+x_{1}^{2} x_{3} x_{4}^{2}+x_{1} x_{2}^{2} x_{3}^{2} \\
& \quad \quad+2 x_{1} x_{2}^{2} x_{3} x_{4}+x_{1} x_{2}^{2} x_{4}^{2}+x_{1} x_{2} x_{3}^{2} x_{4}+x_{1} x_{2} x_{3} x_{4}^{2}+x_{2}^{2} x_{3}^{2} x_{4}+x_{2}^{2} x_{3} x_{4}^{2} .
\end{aligned}
$$

Macdonald [20, 21] showed that when $a$ is weakly increasing of length $n$, we have

$$
\kappa_{a}\left(x_{1}, \ldots, x_{n}\right)=s_{\operatorname{rev}(a)}\left(x_{1}, \ldots, x_{n}\right),
$$

where $\operatorname{rev}(a)$ is the partition obtained by reversing (equivalently, sorting) $a$. In particular, Demazure characters are a polynomial generalization of irreducible characters.

Making this more precise, Demazure crystals are certain subsets of $B(\lambda)$, which were first conjectured by Littelmann [19] to generalize the Demazure characters. This conjecture was later proven by Kashiwara [12]. Given a subset $X \subseteq B(\lambda)$, we define $\mathfrak{D}_{i}$ for $1 \leqslant i<n$ as

$$
\mathfrak{D}_{i} X=\left\{b \in B(\lambda) \mid e_{i}^{k}(b) \in X \text { for some } k \geqslant 0\right\} .
$$

For a permutation $w \in S_{n}$ with reduced expression $w=s_{i_{1}} s_{i_{2}} \cdots s_{i_{k}}$, we define

$$
B_{w}(\lambda)=\mathfrak{D}_{i_{1}} \mathfrak{D}_{i_{2}} \cdots \mathfrak{D}_{i_{k}}\left\{u_{\lambda}\right\}
$$

where $u_{\lambda}$ is the highest weight element in $B(\lambda)$ satisfying $e_{i}\left(u_{\lambda}\right)=0$ for all $1 \leqslant i<n$. Whenever $b, b^{\prime} \in B_{w}(\lambda) \subseteq B(\lambda)$ and $f_{i}(b)=b^{\prime}$ in $B(\lambda)$, then this crystal operator is also defined in $B_{w}(\lambda)$.

Let us define the character of a Demazure crystal as

$$
\operatorname{ch} B_{w}(\lambda)=\sum_{b \in B_{w}(\lambda)} x_{1}^{\mathrm{wt}(b)_{1}} \cdots x_{n}^{\mathrm{wt}(b)_{n}} .
$$

It was proven by $[19,12]$ that this character coincides with $\kappa_{a}$, where $w \cdot a=\lambda$.

\section{Demazure CRYSTAL StRUCTURE ON KEy TABleAuX}

In Section 3.1, we review the combinatorial model of key tableaux [3] that is central to our results. In Section 3.2, we introduce a new crystal structure on semi-standard key tableaux and show that this precisely realizes the Demazure character by truncating the crystal structure on semi-standard Young tableaux.

3.1. Combinatorics of Demazure characters. Combinatorial interpretations and definitions for Demazure characters for the general linear group were given by Lascoux and Schützenberger [17], Kohnert [14], Reiner and Shimozono [25], and Mason [22], all of whom refer to them as key polynomials. We use an equivalent definition in terms of semi-standard key tableaux due to Assaf [3], which is combinatorially equivalent to Mason's semi-skyline augmented fillings but which replaces the triple conditions for more direct row and column conditions (see also [23]). Generalizing Young diagrams, given a weak composition $a$, the key diagram of shape $a$ is the array of left-justified cells with $a_{i}$ boxes in row $i$. 
Definition 3.1 ([3]). A key tableau is a filling of a key diagram with positive integers such that columns have distinct entries, rows weakly decrease, and, if some entry $i$ is above and in the same column as an entry $k$ with $i<k$, then there is an entry immediately right of $k$, say $j$, with $i<j$.

For the Schur polynomial case, we restrict entries in the semi-standard Young tableaux globally allowing entries 1 through $n$ to appear anywhere. In the Demazure case, we must restrict the entries in the semi-standard key tableaux locally allowing entries to appear only in their row and lower.

Definition 3.2 ([1]). A semi-standard key tableau is a key tableau in which no entry exceeds its row index.
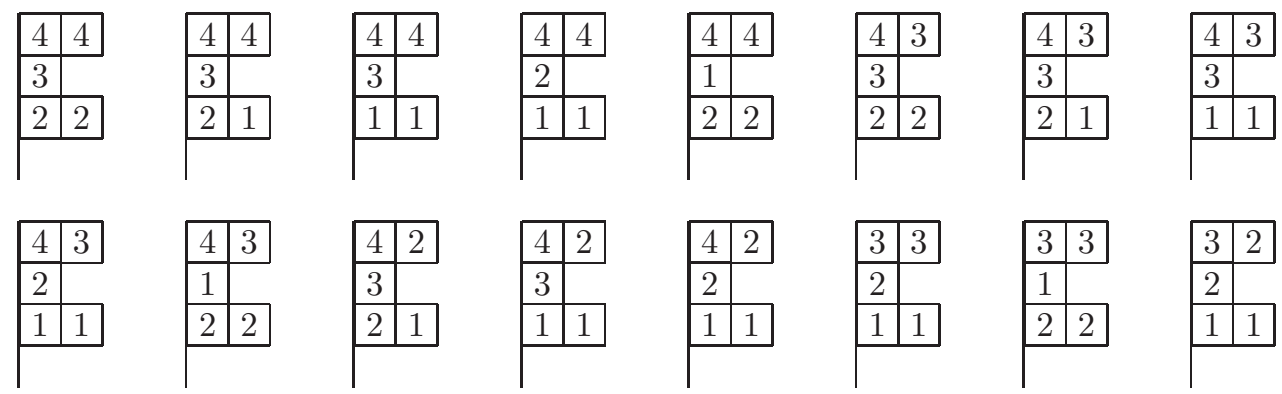

Figure 4. The semi-standard key tableaux of shape $(0,2,1,2)$.

For examples, see Figure 4. The following property of semi-standard key tableaux will be useful.

LEMMA 3.3. Suppose row $r$ of a semi-standard key tableau has two entries $i+1$ in columns $c$ and $c+1$. If there is an $i$ above row $r$ in column $c$, then there cannot be an $i$ below row $r$ in column $c+1$.

Proof. If this were the case, then there must be an entry, say $k$, in column $c$ immediately left of the $i$ in column $c+1$. By the weakly decreasing rows condition, $k \geqslant i$, and so by the distinct column entries condition, and $k>i+1$. However, since there is an $i+1$ above $k$, the entry immediately right of $k$, which is an $i$, is not larger than $i+1$, a contradiction to the key tableaux column inversion condition.

The weight of a semi-standard key tableau $T$, denoted by $\operatorname{wt}(T)$, is the weak composition whose $i$ th part is the number of occurrences of $i$ in $T$. The following result is proved in [1] by showing that the semi-standard key tableaux conditions are equivalent to the triple conditions on Mason's semi-skyline augmented fillings [22]. This more direct characterization facilitates the constructions to follow.

TheOREM 3.4 ([1]). The key polynomial $\kappa_{a}(x)$ is given by

$$
\kappa_{a}(x)=\sum_{T \in \operatorname{SSKT}(a)} x_{1}^{\mathrm{wt}(T)_{1}} \cdots x_{n}^{\mathrm{wt}(T)_{n}},
$$

where $\operatorname{SSKT}(a)$ is the set of semi-standard key tableaux of shape a.

The map from standard key tableaux of shape $a$ to standard Young tableaux of shape $\lambda$, where $\lambda$ is the unique partition rearrangement of $a$, from [3] relates the tableaux models for key polynomials and Schur polynomials. We extend this map to the semi-standard case as follows. 
DEFINITION 3.5. Given a weak composition a of length $n$, define the column sorting map $\phi$ on $\operatorname{SSKT}(a)$ by letting cells fall vertically until there are no gaps between rows, sorting the columns to decrease bottom to top, and then replacing all entries by $i \mapsto n-i+1$.

For example, the semi-standard key tableaux in Figure 4 map to the semi-standard Young tableaux in the first two rows of Figure 1, respectively. The four semi-standard Young tableaux in the bottom row of Figure 1 are not in the image of the column sorting map.

Proposition 3.6. The column sorting map is a well-defined, injective map $\phi: \operatorname{SSKT}(a) \rightarrow \operatorname{SSYT}(\lambda)$, where $\lambda$ is the partition rearrangement of a.

Proof. The distinct column entries condition on semi-standard key tableaux ensures that a column sorted tableau will have strictly increasing columns. By the column inversion condition for key tableaux, if row $j$ sits above row $i$ and is weakly longer, then column by column the entries in row $j$ must be greater than those in row $i$. Consider applying the column sorting map by first rearranging rows of longest size at the bottom and reversing the relative order of rows of equal length. Since entries within rows are maintained, the weakly decreasing row condition on semi-standard key tableaux is obviously maintained by this process. The column sorting necessarily brings entries from a strictly shorter row down into a longer row. That is, row values can be increased only when the first $k$ values all increase for some $k$, and entries decrease only when the entire row is changed, maintaining the weakly decreasing row condition. Hence $\phi(T)$ is indeed a semi-standard Young tableau of shape $\lambda$.

To see that the map is injective, we can define an inverse map by first applying $i \mapsto n-i+1$ to all letters in a semi-standard Young tableau. Then fill the shape of $a$ column by column from right to left, and within a column from bottom to top, according to the columns of the given tableau by selecting at each step the smallest available entry that maintains the weakly decreasing row condition. Since columns are strict in the Young tableau, they will still have distinct entries. It is easy to see that the column inversion condition for key tableaux is maintained, but it could happen that an entry is placed in a row with smaller index. The tableaux for which this occurs are precisely the ones not in the image of the column sorting map.

3.2. Crystal operators on semi-Standard key tableaux. We generalize the crystal structure on semi-standard Young tableaux to a Demazure crystal structure on semi-standard key tableaux as follows.

For a word $w$ of length $k$ with letters in the alphabet $A=\{1,2, \ldots, n\}$, an integer $1 \leqslant r \leqslant k$, and an integer $1 \leqslant i<n$, define

$$
m_{i}(w, r)=\operatorname{wt}\left(w_{r} w_{r+1} \cdots w_{k}\right)_{i+1}-\operatorname{wt}\left(w_{r} w_{r+1} \cdots w_{k}\right)_{i} .
$$

Set $m_{i}(w)=\max _{r}\left\{m_{i}(w, r)\right\}$. Observe that if $m_{i}(w)>0$ and $q$ is the rightmost occurrence of this maximum, then $w_{q}=i+1$, and if $p$ is the leftmost occurrence of this maximum, then either $p=1$ or $w_{p-1}=i$.

For $T$ a key tableau, the column reading word of $T$, denoted by $w(T)$, is the word obtained by reading the entries of $T$ down columns from right to left. Note that columns for key tableaux are read in the reverse order as columns for Young tableaux. For example, the column reading word of the leftmost key tableau in the top row of Figure 4 is 42432 .

DeFinition 3.7. Given an integer $1 \leqslant i<n$, define the raising operators $e_{i}$ on semi-standard key tableaux of shape a of length $n$ as follows: if $m_{i}(w(T)) \leqslant 0$, then $e_{i}(T)=0$; otherwise, let $q$ be the largest index such that $m_{i}(w(T), q)=m_{i}(w(T))$, 
and $e_{i}(T)$ changes all entries $i+1$ weakly right of the entry in $T$ corresponding to $w(T)_{q}$ to $i$ and change all $i$ 's in the same columns as these entries to $i+1$ 's.

Notice that the largest index that attains the maximum must correspond to an $i+1$ and, moreover, that there cannot be an $i$ below it in the same column. Similarly, if there is an $i$ above the $i+1$ in the same column, then there cannot be an $i$ in the column to the left. Moreover, in the case of an $i$ above an $i+1$, there must be an $i+1$ in the column to the right (by the column inversion condition on key tableaux) and both of these $i+1$ s have the same index. In particular, all entries $i+1$ that are changed by $e_{i}$ are in consecutive columns, attain the maximum, and all but the rightmost have an $i$ above them in their column.

For an example of the raising operator $e_{1}$, see Figure 5. For this example, the column reading word is given below each key tableau with the largest index that attains $m_{1}(w(T))>0$ underlined and the corresponding entry in the tableau circled.

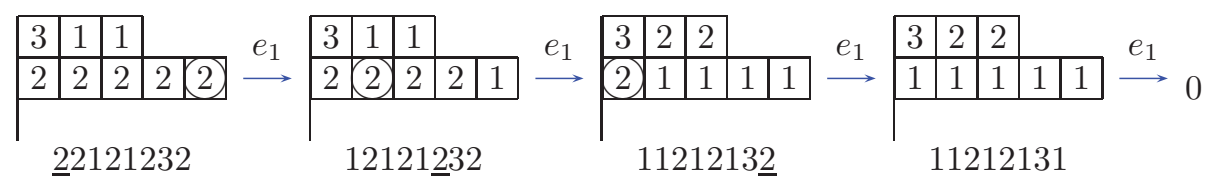

Figure 5. An example of the raising operator $e_{1}$ on key tableaux.

Proposition 3.8. The raising operator $e_{i}: \operatorname{SSKT}(a) \rightarrow \operatorname{SSKT}(a) \cup\{0\}$ is a welldefined map. Moreover, the restriction of $e_{i}$ to the pre-image $e_{i}^{-1}(\operatorname{SSKT}(a))$ satisfies $\operatorname{wt}\left(e_{i}(T)\right)_{i}=\operatorname{wt}(T)_{i}+1, \operatorname{wt}\left(e_{i}(T)\right)_{i+1}=\operatorname{wt}(T)_{i+1}-1$, and $\operatorname{wt}\left(e_{i}(T)\right)_{j}=\operatorname{wt}(T)_{j}$ for all $j \neq i, i+1$.

Proof. Let $T \in \operatorname{SSKT}(a)$, set $m=m_{i}(w(T))$, and suppose $m>0$. Let $x$, say in row $r$ and column $c$, be the cell in $T$ that attains $m$ at the rightmost position in column reading order. We claim that every cell weakly right of $x$ in row $r$ with entry $i+1$ except for one has an $i$ above it. If the entry immediately right of $x$ is $h$ for some $h<i+1$, then the key tableaux conditions ensure that there cannot be an $i$ above $x$ since $h \leqslant i$. Suppose, then, that there is an $i+1$ immediately right of $x$. Since $x$ attains the maximum $m$ and there is an $i+1$ to its left, we must have an $i$ between them in column reading order. Thus there must be an $i$ either below row $r$ in column $c+1$ or above row $r$ in column $c$. If there is an $i$ in row $r^{\prime}<r$ in column $c+1$, then there must be an entry, say $k$, in row $r^{\prime}$ in column $c$ satisfying $k \geqslant i$. Moreover, by the key tableau column inversion condition, we cannot have $k>i+1$ since $i+1>i$. Therefore $k=i$, in which case $x$ cannot be the rightmost position to attain $m$, a contradiction. Moreover, it now follows by induction from Lemma 3.3 that every $i+1$ right of $x$ in row $r$ either has an $i$ above it or no $i$ in its column, and the latter cannot be the case more than once else the rightmost $i+1$ would have $i$-index greater than $m$. This proves the claim, from which it follows that one more cell changes entry from $i+1$ to $i$ than the reverse, thus proving $\operatorname{wt}\left(e_{i}(T)\right)_{i}=\operatorname{wt}(T)_{i}+1, \operatorname{wt}\left(e_{i}(T)\right)_{i+1}=\operatorname{wt}(T)_{i+1}-1$, and $\operatorname{wt}\left(e_{i}(T)\right)_{j}=\operatorname{wt}(T)_{j}$ for all $j \neq i, i+1$.

Next we show that rows of $e_{i}(T)$ are weakly decreasing. This is clear for row $r$ since all $i+1$ weakly right of $x$ change to $i$. If $i$ changes to $i+1$ in cell $y$ and the cell immediately left of $y$ also contains an $i$, then this $i$ also is changed to an $i+1$. This is clear from the previous analysis provided $y$ is not in the column of $x$; if $y$ is in the column of $x$ and has an $i$ immediately to its left, then $x$ cannot be the rightmost cell in column reading order to attain $m$. 
Next we show that columns of $e_{i}(T)$ have distinct entries. Since $x$ cannot have an $i$ below it and be the leftmost cell in column reading order to attain $m$, any $i+1$ that changes to an $i$ either has no $i$ in the column or an $i$ above it. In the latter case, this $i$ will become an $i+1$.

Next we show that in $e_{i}(T)$ if $a<c$ with $a$ above $c$, then there is an entry $b$ immediately right of $c$ with $a<b$. If a column of $T$ contains $i$ and not $i+1$, then nothing is changed, and if it has both, then the $i+1$ appears above $i$ in $e_{i}(T)$. Therefore the only potential problem occurs when $b=i+1$ in $T$ is changed to $i$ in $e_{i}(T)$ and $a=i$. In this case, if the column of $a$ has no $i+1$, then $b$ does not attain $m$ and is not changed to $i$, and otherwise both $a$ and $c$ change removing the inversion triple from consideration.

Finally, decrementing values maintains the property that entries do not exceed their row index, and $i$ changes to $i+1$ only when it sits above an $i+1$, so these entries lie strictly above row $i+1$. Therefore $e_{i}(T)$ is a semi-standard key tableau.

Lemma 3.9. For $T \in \operatorname{SSKT}(a)$ and for any $1 \leqslant i<n, e_{i}(T) \neq 0$ if and only if $f_{n-i}(\phi(T)) \neq 0$. In this case, we have $\phi\left(e_{i}(T)\right)=f_{n-i}(\phi(T))$, where $\phi$ denotes the column sorting map.

Proof. Given a word $w=w_{1} w_{2} \cdots w_{k}$ with $1 \leqslant w_{j} \leqslant n$ for all $j$, let $u=\left(n-w_{k}+\right.$ 1) $\left(n-w_{k-1}+1\right) \cdots\left(n-w_{1}+1\right)$. Then $m_{i}(w, r)=M_{n-i}(u, k-r+1)$, and $q$ is the index of the rightmost occurrence of $m_{i}(w)$ in $w$ if and only if $k-q+1$ is the index of the leftmost occurrence of $M_{n-i}(u)$ in $u$. If $T \in \operatorname{SSKT}(a)$ has no column inversions, then since the column reading word of a semi-standard key tableau is right to left and the column reading word of a semi-standard Young tableau is left to right, $w(T)$ and $w(\phi(T))$ precisely have the relationship of $w$ and $u$, and the result follows.

In the general case, since $e_{i}$ and $f_{i}$ depend only on the letters $i, i+1$, we may restrict our attention to the subword on those letters. In doing so, notice that columns with $i$ above $i+1$ appear in consecutive runs separated at least by a column immediately right of the run with an $i+1$ and no $i$. In the column reading word, this manifests itself as a string of alternating $i$ 's and $i+1$ 's that begins and ends with an $i+1$. If we let $q^{\prime}$ denote the index of the leftmost $i+1$ in the alternating string that attains $m_{i}(w(T))$, then $k-q^{\prime}+1$ is the smallest index that attains $M_{n-i}(w(\phi(T)))$. That is, the rightmost column of $T$ in which an $i+1$ changes to an $i$ without an $i$ also changing to an $i+1$ in passing to $e_{i}(T)$ is precisely the column of $\phi(T)$ in which an $n-i$ changes to an $n-i+1$ in passing to $f_{n-i}(\phi(T))$.

For example, the semi-standard key tableaux of shape $(0,5,3)$ in Figure 5 map by the column sorting map to the semi-standard Young tableaux of shape $(5,3)$ in Figure 2, and the raising operator $e_{1}$ on the former becomes the lowering operator $f_{2}$ on the latter.

Definition 3.10. Given an integer $1 \leqslant i<n$, define the lowering operator $f_{i}$ on semi-standard key tableaux of shape a as follows: let $p$ be the smallest index such that $m_{i}(w(T), p)=m_{i}(w(T))$. If $p=1$ or if the entry in $T$ corresponding to $w_{p}$ lies in row $i$, then $f_{i}(T)=0$; otherwise $f_{i}(T)$ changes all entries $i$ weakly right of the entry in $T$ corresponding to $w_{p-1}$ to $i+1$ and change all $i+1$ 's in the same columns as these entries to $i$ 's.

For examples of lowering operators on semi-standard key tableaux, see Figure 6 ( $f_{i}$ are inverses of $e_{i}$ when they are defined on an element).

Proposition 3.11. For $T \in \operatorname{SSKT}(a)$ and for any $1 \leqslant i<n$, if there exists $S \in$ $\operatorname{SSKT}(a)$ such that $e_{i}(S)=T$, then $f_{i}(T)=S$, and otherwise $f_{i}(T)=0$. In particular, 


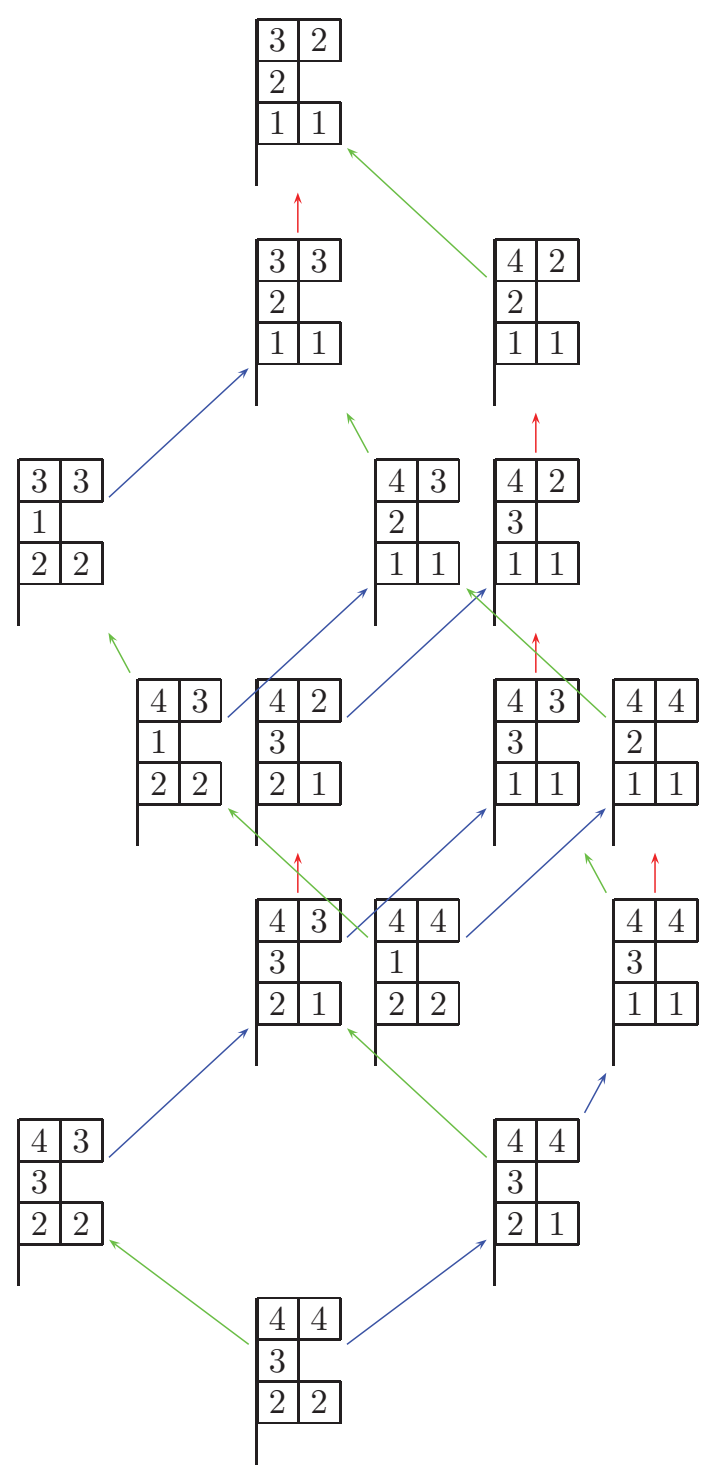

Figure 6. The crystal structure on $\operatorname{SSKT}(0,2,1,2)$, with edges $e_{1} \nearrow, e_{2} \uparrow, e_{3} \nwarrow$.

the lowering operator $f_{i}$ is well-defined and if $f_{i}(T) \neq 0$, then it satisfies $\operatorname{wt}\left(f_{i}(T)\right)_{i}=$ $\mathrm{wt}(T)_{i}+1, \operatorname{wt}\left(f_{i}(T)\right)_{i+1}=\operatorname{wt}(T)_{i+1}-1$, and $\operatorname{wt}\left(f_{i}(T)\right)_{j}=\operatorname{wt}(T)_{j}$ for all $j \neq$ $i, i+1$. Moreover, letting $\phi$ denote the column sorting map, if $f_{i}(T) \neq 0$, then we have $\phi\left(f_{i}(T)\right)=e_{n-i}(\phi(T))$.

Proof. Recall from the analysis in the proof of Lemma 3.9 that when $e_{i}(S) \neq 0, w(S)$ and $w\left(e_{i}(S)\right)$ differ on the restriction to letters $i, i+1$ precisely in that an alternating string beginning and ending with $i+1$ for which the last entry is the rightmost to attain $m_{i}(w(S))$ becomes an alternating string beginning and ending with $i$ for which the first entry is immediately left of the leftmost to attain $m_{i}\left(w\left(e_{i}(S)\right)\right)$. Therefore if $e_{i}(S)=T$, then $f_{i}(T)=S$. We have $f_{i}(T)=0$ precisely when there is no place to act (when $p=1$ ) or when acting would violate the semi-standard key tableaux condition 
that entries cannot exceed their row index. The remainder of the result follows from Proposition 3.8 and Lemma 3.9.

3.3. Demazure CRystal on Semi-Standard Key tableaux. To arrive at our main result, that the raising and lowering operators on semi-standard key tableaux give a Demazure crystal, we refine the column sorting map to an injective map between semi-standard key tableaux for different weak compositions.

LEMMA 3.12. Given a weak composition $a$ and an index $i$ such that $a_{i}<a_{i+1}$, for $T \in \operatorname{SSKT}(a)$ such that $e_{i}(T)=0$, there exists $S \in \operatorname{SSKT}\left(s_{i} a\right)$ such that $\phi(T)=\phi(S)$, where $\phi$ is the column sorting map.

Proof. The statement is equivalent to the assertion that there exists $S \in \operatorname{SSKT}\left(s_{i} a\right)$ with the same column sets as $T$. We may describe the map from $\mathrm{T}$ to $\mathrm{S}$ explicitly as follows. First, move the $a_{i+1}-a_{i}$ rightmost cells in row $i+1$ down to row $i$. Since $e_{i}(T)=0$, there cannot be a letter $i+1$ that is moved down since if any of these cells contain an $i+1$, there will be a positive index allowing $e_{i}$ to act non-trivially. If, after this, row $i$ is not weakly decreasing, then swap the entries in rows $i$ and $i+1$ of the offending columns. Since $e_{i}(T)=0$, there cannot be any letters $i+1$ that are moved down at this step either, so the resulting tableau $S$ has no entry exceeding its row index. Rows clearly maintain their weakly decreasing status, and it is easy to see that no violations of the column inversion condition can arise. Therefore $S \in \operatorname{SSKT}\left(s_{i} a\right)$.

Lemma 3.12 ensures that the following operators are well-defined on semi-standard key tableaux.

DEFINITION 3.13. Given a weak composition a and an index $i$ such that $a_{i}<a_{i+1}$, define an operator $\mathcal{E}_{i}$ on $\operatorname{SSKT}(a)$ by $\mathcal{E}_{i}(T)=S$, where $S \in \operatorname{SSKT}\left(s_{i} a\right)$ satisfies $\phi(S)=\phi\left(e_{i}^{k-1}(T)\right)$ for $k$ minimal such that $e_{i}^{k}(T)=0$.

For examples of $\mathcal{E}_{i}$, see Figure 7 . Similar to $\pi_{w}$ and $\partial_{w}$, we may extend this to define $\mathcal{E}_{w}=\mathcal{E}_{i_{1}} \cdots \mathcal{E}_{i_{k}}$, where $s_{i_{1}} \cdots s_{i_{k}}$ is any reduced expression for $w$. It is easy to see that this is well-defined from the local relations of the type $A$ crystal operators on tableaux as characterized by Stembridge [28].

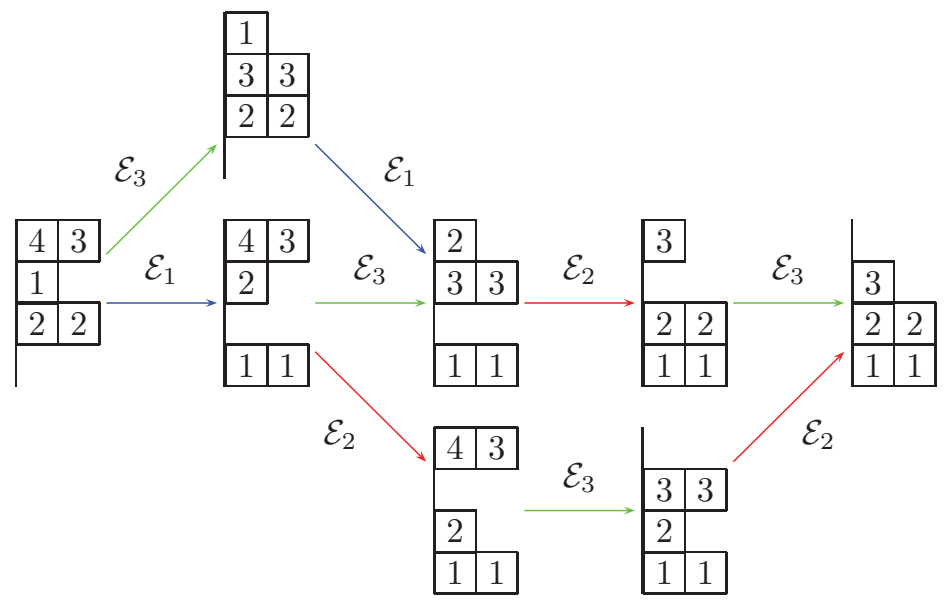

Figure 7. An example of the $\mathcal{E}_{i}$ operators on semi-standard key tableaux. 
Given a weak composition $a$, for $w$ the permutation that sorts $a$ to partition shape $\lambda$, the operator $\mathcal{E}_{w}$ takes $T \in \operatorname{SSKT}(a)$ to the highest weight element of the crystal along edges specified by $w$. This is precisely the statement needed to show that the crystal operators defined on semi-standard key tableaux of shape $a$ realize the Demazure crystal for $w$.

THEOREM 3.14. Let a be a weak composition that sorts to the partition $\lambda$. The raising and lowering operators on $\operatorname{SSKT}(a)$ give the Demazure crystal for highest weight $\lambda$ truncating with respect to the minimal length permutation $w$ that sorts a to $\lambda$.

Proof. Given $T \in \operatorname{SSKT}(a)$, for $w$ the permutation that sorts $a$ to partition shape $\lambda$, we necessarily have $\mathcal{E}_{w}(T) \in \operatorname{SSKT}(\lambda)$. However, the constraint that entries cannot exceed their row index together with distinct column values forces $\operatorname{SSKT}(\lambda)$ to have a single element, the tableau with all entries in row $i$ equal to $i$. In particular, this element maps via the column sorting map to the highest weight $u_{\lambda}$. By Lemma 3.9, this means $\phi(T) \in \mathfrak{D}_{w}\left\{u_{\lambda}\right\}$ for every $T \in \operatorname{SSKT}(a)$, and so $\phi(\operatorname{SSKT}(a)) \subseteq B_{w}(\lambda)$. By Theorem 3.4, the sums of the weights on both sides agree, so we must have equality.

For example, removing the four vertices of the $(2,2,1)$-crystal in Figure 3 corresponding to the four semi-standard Young tableaux of shape $(2,2,1)$ that are not in the image of the column sorting map on semi-standard key tableaux of shape $(0,2,1,2)$ precisely gives the $(0,2,1,2)$-Demazure crystal in Figure 6 .

\section{Crystal structure for Stanley symmetric polynomials}

We review the combinatorics of Stanley symmetric functions and polynomials in terms of reduced factorizations of a permutation in Section 4.1. We proceed in Section 4.2 to review Edelman-Greene insertion and review the crystal structure on reduced factorizations as recently introduced in [24] in Section 4.3.

4.1. Combinatorics of Stanley Symmetric functions. Stanley [27] introduced a new family of symmetric functions to enumerate reduced expressions for permutations.

Definition 4.1. A reduced word for a permutation $w \in S_{n}$ is a word $i_{1} \ldots i_{k}$ such that $s_{i_{1}} \cdots s_{i_{k}}=w$ where $k$ is the inversion number of $w$. Denote the set of reduced words for $w$ by $R(w)$.

For example, there are 11 reduced words for the permutation 153264 as shown in Figure 8.

$$
4532345232435234253243253245324235243235243524232524325
$$

Figure 8. The reduced words for 153264 .

DEFINITION 4.2. Given a reduced word $\rho$, an increasing factorization for $\rho$ partitions the word $\rho$ into (possibly empty) blocks (or factors) such that entries increase left to right within each block.

Given a permutation $w$, a reduced factorization for $w$ is an increasing factorization of a reduced word for $w$. Denote the set of reduced factorizations for $w$ by $\operatorname{RF}(w)$. 


$\begin{array}{lr}()(45)(3)(23)()(45)(23)(2)()(4)(35)(23)(4)(25)(3)(2)(4)(3)(25)(3) & ()(245)(3)(2) \\ (4)(5)(3)(23)(4)(5)(23)(2)(4)()(35)(23) & (2)(45)(3)(2) \\ (45)()(3)(23)(45)()(23)(2)(4)(3)(5)(23) & (24)(5)(3)(2) \\ (45)(3)()(23)(45)(2)(3)(2)(4)(35)()(23) & (245)()(3)(2) \\ (45)(3)(2)(3)(45)(23)()(2)(4)(35)(2)(3) & (245)(3)()(2) \\ (45)(3)(23)()(45)(23)(2)()(4)(35)(23)() & (245)(3)(2)()\end{array}$

()$(4)(235)(2)()(4)(3)(235)()(24)(35)(2)()(4)(23)(25)()(24)(3)(25)$

$(4)()(235)(2)(4)()(3)(235)(2)(4)(35)(2)(4)()(23)(25)(2)(4)(3)(25)$

$(4)(2)(35)(2)(4)(3)()(235)(24)()(35)(2)(4)(2)(3)(25)(24)()(3)(25)$

$(4)(23)(5)(2)(4)(3)(2)(35)(24)(3)(5)(2)(4)(23)()(25)(24)(3)()(25)$

$(4)(235)()(2)(4)(3)(23)(5)(24)(35)()(2)(4)(23)(2)(5)(24)(3)(2)(5)$

$(4)(235)(2)()(4)(3)(235)()(24)(35)(2)()(4)(23)(25)()(24)(3)(25)()$

Figure 9. The reduced factorizations for 153264 into 4 blocks.

For example, the reduced factorizations for 153264 into 4 blocks are shown in Figure 9.

The weight of a reduced factorization $r$, denoted by $\operatorname{wt}(r)$, is the weak composition whose $i$ th part is the number of letters in the $i$ th block of $r$ from the right. For example, $\operatorname{wt}((45)(3)(23)())=(0,2,1,2)$.

Definition 4.3. The Stanley symmetric function indexed by the permutation $w$ is

$$
F_{w}(x)=\sum_{r \in \operatorname{RF}\left(w^{-1}\right)} x^{\mathrm{wt}(r)} .
$$

Therefore we compute $F_{143625}$ using reduced factorizations for $143625^{-1}=153264$.

Note that reduced factorizations can, in principle, have an arbitrary number of blocks and hence $F_{w}(x)$ is a symmetric function in infinitely many variables $x=$ $\left(x_{1}, x_{2}, \ldots\right)$.

We can restrict Stanley symmetric functions to Stanley symmetric polynomials by restricting the number of blocks in the reduced factorizations. Let $\operatorname{RF}^{\ell}(w)$ be the set of reduced factorizations of $w$ with precisely $\ell$ blocks. Then the Stanley symmetric polynomial in $\ell$ variables is

$$
F_{w}\left(x_{1}, x_{2}, \ldots, x_{\ell}\right)=\sum_{r \in \mathrm{RF}^{\ell}\left(w^{-1}\right)} x^{\mathrm{wt}(r)} .
$$

4.2. Edelman-Greene CORRESPONDEnCE. In their study of Stanley symmetric functions, Edelman and Greene [10] developed the following insertion algorithm that they used to give a formula for the Schur expansion of Stanley symmetric functions.

Definition 4.4 ([10, Definition 6.21]). Let $P$ be a Young tableau, and let $x$ be a positive integer. Let $P_{i}$ be the ith lowest row of $P$. Define the Edelman-Greene insertion of $x$ into $P$, denoted by $P \leftarrow x$, as follows. Set $x_{0}=x$ and for $i \geqslant 0$, insert $x_{i}$ into $P_{i+1}$ as follows. If $x_{i} \geqslant z$ for all $z \in P_{i+1}$, place $x_{i}$ at the end of $P_{i+1}$ and stop. Otherwise, let $x_{i+1}$ denote the smallest element of $P_{i+1}$ such that $x_{i+1}>x_{i}$. If $x_{i+1} \neq x_{i}+1$ or $x_{i}$ is not already in $P_{i+1}$, replace $x_{i+1}$ by $x_{i}$ in $P_{i+1}$ and continue (we say that $x_{i}$ bumps $x_{i+1}$ in row $\left.i+1\right)$. Otherwise leave $P_{i+1}$ unchanged and continue with $x_{i+1}$.

Given a reduced expression $\rho$, define the insertion tableau for $\rho$, denoted by $P(\rho)$, to be the result of inserting the word for $\rho$ letter by letter into the empty tableau. 
To track the growth of $P(\rho)$, define the recording tableau for $\rho$, denoted by $Q(\rho)$, to be the result of adding $i$ into the new cell created when inserting the $i$ th letter. For example, Figure 10 constructs the insertion tableau (top) and recording tableau (bottom) for the reduced expression 45232.

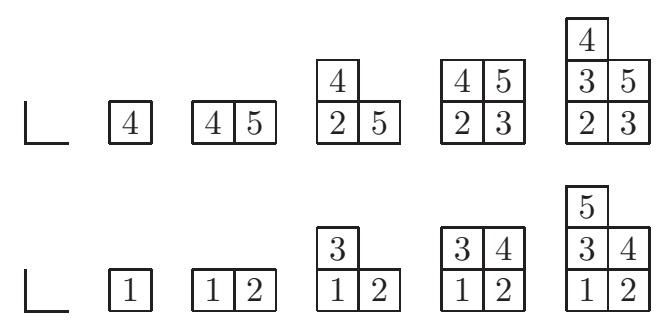

FIGURE 10. The insertion and recording tableaux for the reduced expression 45232 .

ThEOREM 4.5 ([10, Theorem 6.25]). The Edelman-Greene correspondence $\rho \mapsto$ $(P(\rho), Q(\rho))$ is a bijection between reduced expressions and all pairs of tableaux $(P, Q)$ such that $P$ and $Q$ have the same shape, $P$ is increasing with $\operatorname{row}(P)$ a reduced word, and $Q$ is standard.

We may extend the Edelman-Greene correspondence to a bijection between reduced factorizations and all pairs of tableaux $(P, Q)$ such that $P$ and $Q$ have the same shape, $P$ is increasing with $\operatorname{row}(P)$ a reduced word, and $Q$ is semi-standard. To do so, given a reduced factorization $r$ into $\ell$ blocks, define $P(r)$ to be $P(\rho)$ where $\rho$ is the underlying reduced expression for $r$, and define $Q(r)$ to be the result of adding $\ell-i+1$ into each new cell created when inserting a letter from block $i$ (from the right). For example, the recording tableau for the reduced factorization $(4)(5)(23)(2)$ is constructed in Figure 11.

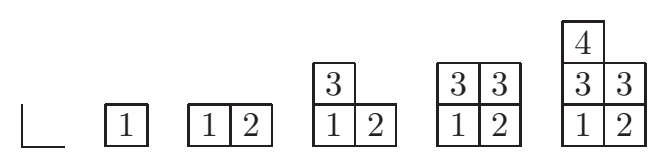

FIGURE 11. The recording tableau for the reduced factorization $(4)(5)(23)(2)$.

COROLlary 4.6. The correspondence $r \mapsto(P(r), Q(r))$ is a bijection between reduced factorizations and all pairs of tableaux $(P, Q)$ such that $P$ and $Q$ have the same shape, $P$ is increasing with $\operatorname{row}(P)$ a reduced word, and $Q$ is semi-standard. Moreover, if $r$ has $\ell$ blocks, then $\operatorname{wt}(Q(r))_{i}=\operatorname{wt}(r)_{\ell-i+1}$.

For example, the Edelman-Greene correspondence gives a weight-reversing bijection

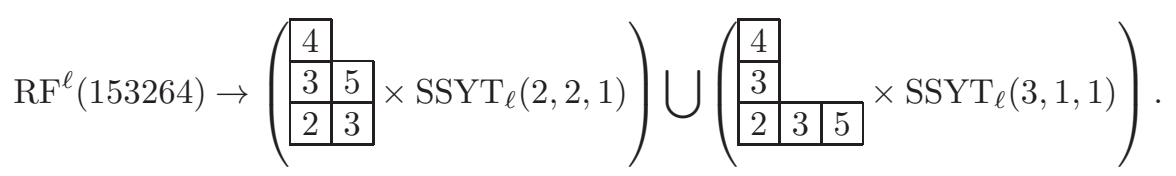

In particular, by the symmetry of Schur functions, we have the following expansion from [10]. 
Corollary 4.7. The Stanley symmetric function for $w$ may be expressed as

$$
F_{w}(x)=\sum_{T \in \operatorname{Yam}\left(w^{-1}\right)} s_{\operatorname{sh}(T)}(x),
$$

where $\operatorname{Yam}\left(w^{-1}\right)$ is the set of insertion tableaux with $\operatorname{row}(P)$ a reduced word for $w^{-1}$.

For example, we have

$$
F_{143625}(x)=s_{(2,2,1)}(x)+s_{(3,1,1)}(x) .
$$

4.3. Crystal operators on Reduced FaCtorizations. Following [24], we are going to define an $A_{\ell-1}$-crystal structure on $\mathrm{RF}^{\ell}(w)$. Let $r=r^{\ell} r^{\ell-1} \cdots r^{1} \in \mathrm{RF}^{\ell}(w)$, where $r^{i}$ is the $i$ th block from the right. The Kashiwara raising and lowering operators $e_{i}$ and $f_{i}$ only act on the blocks $r^{i+1} r^{i}$. The action is defined by first bracketing certain letters and then moving an unbracketed letter from one factor to the other. Let us begin by describing the bracketing procedure. Start with the largest letter $b$ in $r^{i}$ and pair it with the smallest $a>b$ in $r^{i+1}$. If no such $a$ exists in $r^{i+1}$, then $b$ is unpaired. The pairing proceeds in decreasing order on elements of $r^{i}$, and with each iteration previously paired letters of $r^{i+1}$ are ignored. Define

$$
R_{i}\left(r^{\ell} \cdots r^{1}\right)=\left\{b \in r^{i} \mid b \text { is unpaired in the } r^{i+1} r^{i} \text {-pairing }\right\}
$$

and

$$
L_{i}\left(r^{\ell} \cdots r^{1}\right)=\left\{b \in r^{i+1} \mid b \text { is unpaired in the } r^{i+1} r^{i} \text {-pairing }\right\} .
$$

Then $f_{i}\left(r^{\ell} \cdots r^{1}\right)$ is defined by replacing the blocks $r^{i+1} r^{i}$ by $\widetilde{r}^{i+1} \widetilde{r}^{i}$ such that

$$
\widetilde{r}^{i}=r^{i} \backslash\{b\} \quad \text { and } \quad \tilde{r}^{i+1}=r^{i+1} \cup\{b-t\}
$$

for $b=\min \left(R_{i}\left(r^{\ell} \cdots r^{1}\right)\right)$ and $t=\min \left\{j \geqslant 0 \mid b-j-1 \notin r^{i}\right\}$. If $R_{i}\left(r^{\ell} \cdots r^{1}\right)=\varnothing$, then $f_{i}\left(r^{\ell} \cdots r^{1}\right)=0$.

Similarly, $e_{i}\left(r^{\ell} \cdots r^{1}\right)$ is defined by replacing the factors $r^{i+1} r^{i}$ by $\widetilde{r}^{i+1} \widetilde{r}^{i}$ such that

$$
\widetilde{r}^{i}=r^{i} \cup\{a+s\} \quad \text { and } \quad r^{i+1}=r^{i+1} \backslash\{a\}
$$

for $a=\max \left(L_{i}\left(r^{\ell} \cdots r^{1}\right)\right)$ and $s=\min \left\{j \geqslant 0 \mid a+j+1 \notin r^{i+1}\right\}$. If $L_{i}\left(r^{\ell} \cdots r^{1}\right)=\varnothing$, then $e_{i}\left(r^{\ell} \cdots r^{1}\right)=0$.

EXAMPLE 4.8. Let $(2)(13)(23) \in \mathrm{RF}^{3}(w)$ for $w=s_{2} s_{1} s_{3} s_{2} s_{3} \in S_{4}$. To apply $f_{1}$ we need to first bracket the letters in $r^{1}=23$ with those in $r^{2}=13$. The letter 3 in $r^{1}$ is unbracketed since there is no bigger letter in $r^{2}$, but the letter 2 in $r^{1}$ is bracketed with 3 in $r^{2}$. Hence $b=\min \left(R_{1}\left(r^{3} r^{2} r^{1}\right)\right)=3$ and $t=\min \left\{j \geqslant 0 \mid b-j-1 \notin r^{1}\right\}=1$. Therefore, $f_{1}((2)(13)(23))=(2)(123)(2)$. Similarly, $e_{1}((2)(13)(23))=(2)(3)(123)$.

REMARK 4.9. In [24], the Stanley symmetric function $F_{w}$ is defined using decreasing factorizations of reduced words of $w$. Here we use increasing factorizations of $w^{-1}$. To relate the two, one needs to revert the reduced factorizations. The crystal structures are related by interchanging $f_{i}$ (resp. $e_{i}$ ) with $e_{\ell-i}$ (resp. $f_{\ell-i}$ ).

ThEOREM 4.10 ([24, Theorem 3.5]). The above defined operators $f_{i}$ and $e_{i}$ for $1 \leqslant i<$ $\ell$ and the weight function wt define a $A_{\ell-1}$-crystal structure on $\mathrm{RF}^{\ell}(w)$.

COROLlary 4.11 ([24]). The Stanley symmetric function for $w$ may be expressed as

$$
F_{w}(x)=\sum_{\substack{r \in \mathrm{RF}^{\ell}\left(w^{-1}\right) \\ e_{i} r=0}} s_{\mathrm{wt}(r)}(x) .
$$


For example, the highest weight reduced factorizations for $153264=143625^{-1}$ with $\ell=4$ are ()$(4)(35)(23)$ and ()$(4)(3)(235)$ of weights $(2,2,1)$ and $(3,1,1)$, respectively, confirming (4.3).

It turns out that this crystal structure on reduced factorizations relates to the crystal structure on semi-standard Young tableaux via the Edelman-Greene correspondence.

Theorem 4.12 ([24, Theorem 4.11]). Given $r \in \mathrm{RF}^{\ell}(w)$, let $P(r)$ denote its Edelman-Greene insertion tableau and $Q(r)$ its Edelman-Greene semi-standard recording tableau, where letters in block $i$ of $r$ are recorded by the letter $i$. Then, if $e_{i}(r) \neq 0$, we have $P\left(e_{i}(r)\right)=P(r)$ and $Q\left(e_{i}(r)\right)=f_{\ell-i}(Q(r))$.

\section{Demazure Crystal Structure for Schubert polynomials}

We review the combinatorial expression of Billey, Jockusch and Stanley [7] for Schubert polynomials in terms of compatible sequences in Section 5.1 and show that it can be reformulated in terms of reduced factorizations with a cutoff condition. In Section 5.2 we discuss the weak analog of the Edelman-Greene insertion presented in [2]. It turns out that the cut-off condition precisely amounts to a Demazure crystal structure as shown in Section 5.3.

5.1. Combinatorics of Schubert polynomials. Schubert polynomials are generalizations of Schur polynomials which represent cohomology classes of Schubert cycles in flag varieties. They were first introduced by Bernstein et al. [6] and extensively studied by Lascoux and Schützenberger [15].

DEFINITION 5.1 ([15]). Given a permutation $w$, the Schubert polynomial for $w$ is given by

$$
\mathfrak{S}_{w}(x)=\partial_{w^{-1} w_{0}}\left(x_{1}^{n-1} x_{2}^{n-2} \cdots x_{n-1}\right),
$$

where $w_{0}=n n-1 \ldots 21$ is the longest permutation of length $\left(\begin{array}{l}n \\ 2\end{array}\right)$.

The first proven combinatorial formula for Schubert polynomials, due to Billey, Jockusch and Stanley [7], is in terms of compatible sequences for reduced expressions.

DEFINITION $5.2([7])$. For $\rho=\rho_{1} \ldots \rho_{k}$ a reduced word, a sequence $\alpha=\alpha_{1} \ldots \alpha_{k}$ of positive integers is $\rho$-compatible if $\alpha$ is weakly decreasing, $\alpha_{j} \leqslant \rho_{j}$, and $\alpha_{j}>\alpha_{j+1}$ whenever $\rho_{j}>\rho_{j+1}$. Denote the set of compatible sequences for the reduced word $\rho$ by $R C(\rho)$.

For example, seven of the reduced words for 153264 have compatible sequences as shown in Figure 12.

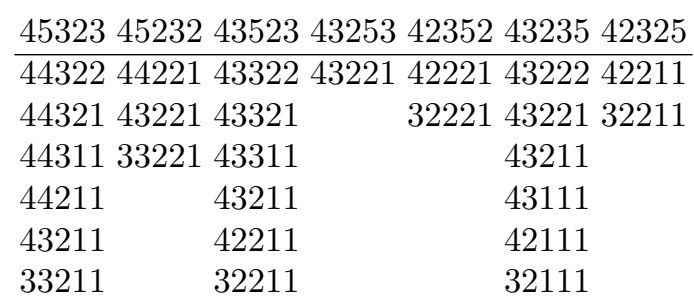

FiguRE 12. The compatible sequences for the reduced words for 153264 . 
Theorem $5.3([7])$. The Schubert polynomial $\mathfrak{S}_{w}(x)$ indexed by a permutation $w$ is given by

$$
\mathfrak{S}_{w}(x)=\sum_{\rho \in R\left(w^{-1}\right)} \sum_{\alpha \in \operatorname{RC}(\rho)} x_{\alpha},
$$

where $x_{\alpha}$ is the monomial $x_{\alpha_{1}} \cdots x_{\alpha_{n}}$.

We may encode compatible sequences for the reduced words as increasing factorizations with an additional cutoff condition.

DEFINITION 5.4. Given a reduced word $\rho$, an increasing factorization with cutoff is an increasing factorization such that in addition the first entry in block $i$ from the right is at least $i$.

Given a permutation $w$, a reduced factorization with cutoff for $w$ is an increasing factorization with cutoff of a reduced word for $w$.

The set of reduced factorizations with cutoff is denoted by $\operatorname{RFC}(w)$. For example, the reduced factorizations with cutoff for 153264 are shown in Figure 13.

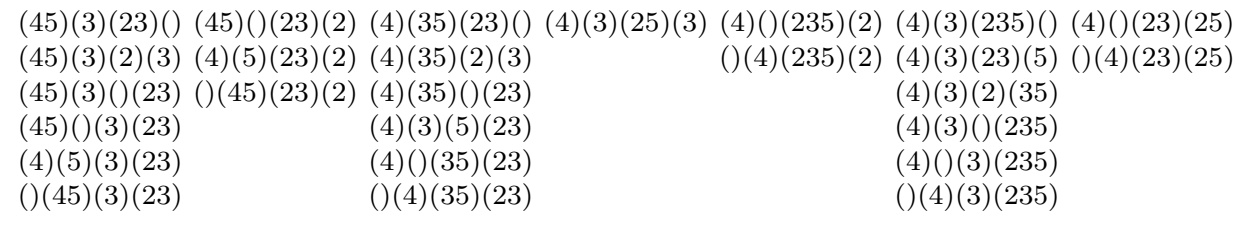

FIGURE 13. The reduced factorizations with cutoff for 153264 .

The weight function on reduced factorizations provides a simple bijection between compatible sequences and increasing factorizations with cutoff for a reduced word. For example, compare Figure 13 with Figure 12.

Proposition 5.5. The Schubert polynomial $\mathfrak{S}_{w}(x)$ is given by

$$
\mathfrak{S}_{w}(x)=\sum_{r \in \operatorname{RFC}\left(w^{-1}\right)} x^{\mathrm{wt}(r)} .
$$

Proof. To prove that (5.3) is equivalent to (5.2), we show that there is a bijection $\bigcup_{\rho \in R\left(w^{-1}\right)} \operatorname{RC}(\rho) \rightarrow \operatorname{RFC}\left(w^{-1}\right)$ that preserves the weights. Given a compatible sequence $\alpha$ for a reduced word $\rho$, the letter $\rho_{i}$ belongs to the $a$-th factor from the right if $\alpha_{i}=a$. Due to the condition that $\alpha_{j}>\alpha_{j+1}$ whenever $\rho_{j}>\rho_{j+1}$, the letters within each factor are weakly increasing. Since the word $\rho$ is reduced, the letters within each factor must actually be increasing. Furthermore, since $\alpha_{j} \leqslant \rho_{j}$, all letters in the $a$-th factor must be of value at least $a$. Conversely, given a reduced factorization with cutoff one can immediately construct the compatible sequence $\alpha$ by setting $\alpha_{j}=a$ if $\rho_{j}$ is in factor $a$.

Reduced factorizations have the advantage of tracking the reduced word along with the weight, making this a more natural indexing set for the crystal structure discussed in the next section.

5.2. Weak Edelman-Greene CORRESPondence. We recall a generalization of the Edelman-Greene correspondence [2] that gives the Demazure expansion of a Schubert polynomial, parallel to the Schur expansion of a Stanley symmetric function.

Following [2], for $P$ a semi-standard Young tableau with strictly increasing rows, define the lift of $P$, denoted by $\operatorname{lift}(P)$, to be the tableau of key shape obtained by 
raising each cell in the first column of $P$ until its entry equals its row index, and, once columns 1 through $c-1$ have been lifted, raising cells in column $c$ from top to bottom, maintaining their relative order, placing each cell in the highest available row such that there is an entry in column $c-1$ that is strictly smaller.

DEFinition 5.6 ([2]). For $\rho$ a reduced expression, define the weak insertion tableau $\widehat{P}(\rho)$ by $\widehat{P}(\rho)=\operatorname{lift}(P(\rho))$, where $P(\rho)$ is the insertion tableau under the EdelmanGreene insertion. In addition, define the weak recording tableau $\widehat{Q}(\rho)$ to be the unique standard key tableau of the same key shape as $\widehat{P}(\rho)$ such that $\phi(\widehat{Q}(\rho))=Q(\rho)$, where $Q(\rho)$ is the Edelman-Greene recording tableau and $\phi$ is the column sorting map.

For example, Figure 14 constructs the weak insertion tableau (top) and weak recording tableau (bottom) for the reduced expression 45232. Compare this with Figure 10.

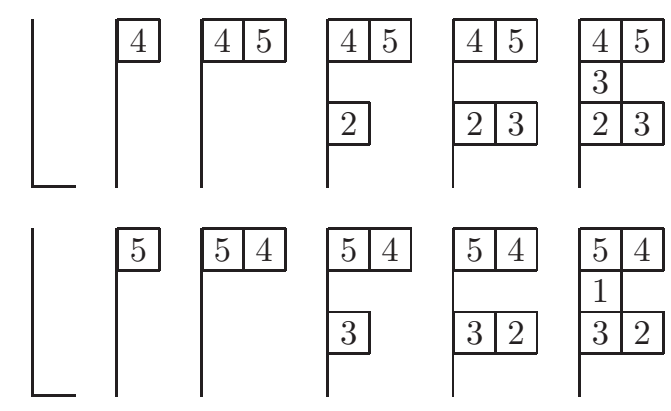

FiguRE 14. The weak insertion and recording tableaux for the reduced expression 45232 .

For $P$ a key tableau, define the drop of $P$, denoted by $\operatorname{drop}(P)$, to be the Young tableau defined by letting the entries of $P$ fall in their columns while maintaining their relative order. It is clear that $\operatorname{drop}(\operatorname{lift}(P))=P$ for any $P$ of partition shape.

THEOREM 5.7 ([2]). The weak Edelman-Greene correspondence $\rho \mapsto(\widehat{P}(\rho), \widehat{Q}(\rho))$ is a bijection between reduced expressions and all pairs of tableaux $(P, Q)$ such that $P$ and $Q$ have the same key shape, $P$ is increasing (in rows and columns) with $\operatorname{row}(P)$ a reduced word and $\operatorname{lift}(\operatorname{drop}(P))=P$, and $Q$ is a standard key tableau.

Analogous to the Edelman-Greene correspondence, this extends to a bijection between reduced factorizations with cutoff and all pairs of tableaux $(P, Q)$ such that $P$ and $Q$ have the same key shape, $P$ is increasing with $\operatorname{row}(P)$ a reduced word and $\operatorname{lift}(\operatorname{drop}(P))=P$, and $Q$ is a semi-standard key tableau. For example, the recording tableau for the reduced factorization $(4)(5)(23)(2)$ is constructed in Figure 15.

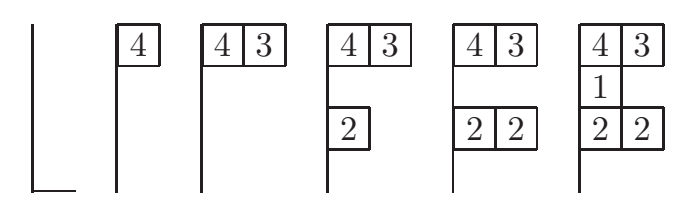

FigurE 15. The weak recording tableau for the reduced factorization $(4)(5)(23)(2)$. 
COROLLARY 5.8. The correspondence $r \mapsto(\widehat{P}(r), \widehat{Q}(r))$ is a weight-preserving bijection between reduced factorizations with cutoff and all pairs of tableaux $(P, Q)$ such that $P$ and $Q$ have the same key shape, $P$ is increasing with $\operatorname{row}(P)$ a reduced word and $\operatorname{lift}(\operatorname{drop}(P))=P$, and $Q$ is a semi-standard key tableau.

Proof. Theorem 5.7 is proved in [2, Theorem 5.16] using the standard key tableau. To get the semi-standard case, we appeal to [1, Proposition 2.6] where it is shown that the fundamental slide polynomial, defined in [4], associated to a standard key tableau is the sum of monomials associated to the semi-standard key tableaux that standardize to it. As shown in [2, Theorem 2.4], the fundamental slide polynomial associated to a reduced expression is the sum of monomials associated to the corresponding compatible sequences. The result follows from the bijection between compatible sequences and increasing factorizations with cutoff.

For example, the weak Edelman-Greene correspondence gives a weight-preserving bijection

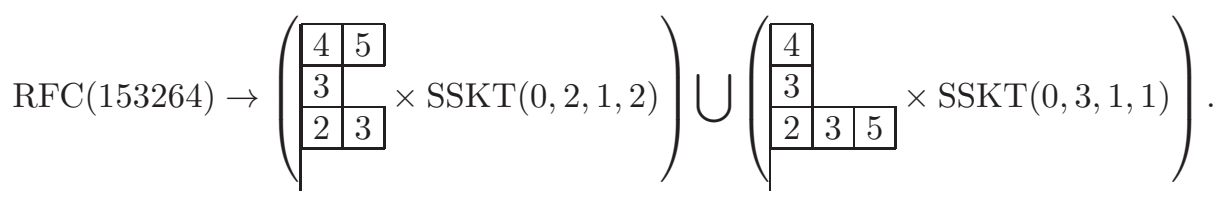

In particular, we have the following expansion from [2].

CoROLlary 5.9 ([2]). The Schubert polynomial for $w$ may be expressed as

$$
\mathfrak{S}_{w}(x)=\sum_{T \in \operatorname{Yam}\left(w^{-1}\right)} \kappa_{\mathrm{wt}(T)}(x),
$$

where $\operatorname{Yam}\left(w^{-1}\right)$ is the set of increasing tableaux of key shape with $\operatorname{row}(P)$ a reduced word for $w^{-1}$ and $\operatorname{lift}(\operatorname{drop}(P))=P$.

For example, we have

$$
\mathfrak{S}_{143625}(x)=\kappa_{(0,2,1,2)}(x)+\kappa_{(0,3,1,1)}(x) .
$$

5.3. DemazURE CRYSTAL OPERATORS ON REDUCED FACTORIZATIONS With CUTOFF. Since $\operatorname{RFC}(w) \subseteq \operatorname{RF}^{n}(w)$ for $w \in S_{n}$, we can restrict the crystal operators $f_{i}$ and $e_{i}$ on reduced factorizations to $\operatorname{RFC}(w)$ by defining $f_{i}(r)$ as in Section 4.3 if $f_{i}(r) \in \operatorname{RFC}(w)$ and $f_{i}(r)=0$ otherwise and similarly for $e_{i}$. An example is given in Figure 16 .

We will show in this section that this amounts to a union of Demazure crystal structures. We begin with an analog of Theorem 4.12.

Theorem 5.10. Given $r \in \mathrm{RFC}(w)$ for $w \in S_{n}$, denote by $\widehat{P}(r)$ the weak EdelmanGreene insertion tableau and by $\widehat{Q}(r)$ the weak Edelman-Greene recording tableau, where letters in block $i$ of $r$ are recorded by the letter $i$. Then, if $e_{i}(r) \neq 0$, we have $\widehat{P}\left(e_{i}(r)\right)=\widehat{P}(r)$ and $\widehat{Q}\left(e_{i}(r)\right)=e_{i}(\widehat{Q}(r))$ for $1 \leqslant i<n$.

Proof. By Theorem 4.12 we have $P\left(e_{i}(r)\right)=P(r)$ and $Q\left(e_{i}(r)\right)=f_{n-i}(Q(r))$, where $P$ and $Q$ are the Edelman-Greene insertion and recording tableaux, respectively. By Definition 5.6, we have $\widehat{P}(r)=\operatorname{lift}(P(r))$, which proves $\widehat{P}\left(e_{i}(r)\right)=\widehat{P}(r)$. Again by Definition 5.6, we have $\phi(\widehat{Q}(r))=Q(r)$. By Lemma 3.9, we have $\phi\left(e_{i} \widehat{Q}(r)\right)=$ $f_{n-i} \phi(\widehat{Q}(r))=f_{n-i} Q(r)$, proving that $\widehat{Q}\left(e_{i}(r)\right)=e_{i}(\widehat{Q}(r))$.

By Proposition 5.5, combinatorial objects underlying the Schubert polynomials $\mathfrak{S}_{w^{-1}}(x)$ are the reduced factorizations with cutoff $\operatorname{RFC}(w)$. On the other hand, $\mathrm{RF}^{n}(w)$ are combinatorial objects underlying the Stanley symmetric polynomials 

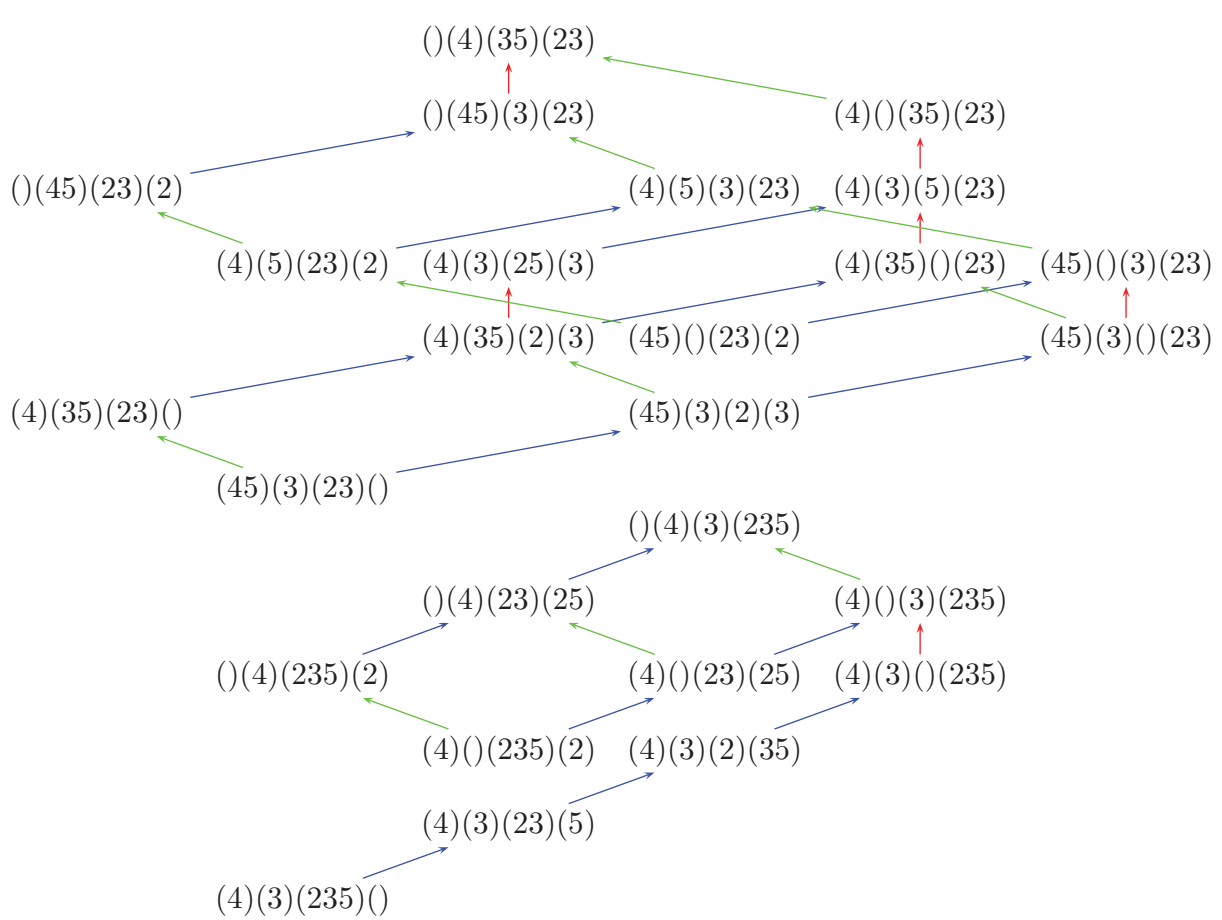

FIGURE 16. The Demazure crystal structure on RFC(153264), with edges $e_{1} \nearrow, e_{2} \uparrow, e_{3} \nwarrow$.

$F_{w^{-1}}(x)$ by Definition 4.3. By Theorem 4.10, there is a crystal structure on $\operatorname{RF}^{n}(w)$. Now we show that $\operatorname{RFC}(w)$ admits a Demazure crystal structure.

THEOREM 5.11. The operators $f_{i}$ and $e_{i}$ for $1 \leqslant i<n$ define a Demazure crystal structure on $\operatorname{RFC}(w)$. More precisely,

$$
\mathrm{RFC}(w) \cong \bigcup_{\substack{r \in \mathrm{RFCC}(w) \\ e_{i} r=0}} B_{w(r) i<n}(\operatorname{wt}(r)),
$$

where $w(r)$ is the shortest permutation that sorts $\operatorname{sh}(\widehat{P}(r))$.

Proof. By Theorem 5.10, the crystal operators on reduced factorizations under weak Edelman-Greene insertion intertwine with the crystal operators on key tableaux. On the other hand, by Theorem 3.14 the crystal operators on key tableaux form a Demazure crystal.

For example, the highest weight elements in $\operatorname{RFC}(153264)$ are ()$(4)(35)(23)$ and ()(4)(3)(235) (see Figure 16), so that as Demazure crystals

$$
\mathrm{RFC}(153264) \cong B_{s_{1} s_{3} s_{2} s_{3}}(2,2,1) \cup B_{s_{1} s_{2} s_{3}}(3,1,1) .
$$

COROLlaRY 5.12. The Schubert polynomial for $w \in S_{n}$ may be expressed as

$$
\mathfrak{S}_{w}(x)=\sum_{\substack{r \in \operatorname{RFC}\left(w^{-1}\right) \\ e_{i} r=0}}^{\forall 1 \leqslant i<n} \underset{\operatorname{sh}(\widehat{P}(r))}{ }(x) .
$$

Acknowledgements. The authors are grateful to Per Alexandersson, Sara Billey, Jim Haglund, Cristian Lenart, Sarah Mason, Liz Milicevic, Jennifer Morse, Vic Reiner, Mark Shimozono, and Alex Yong for helpful discussions and comments on this topic. 
AS would also like to thank the University of Southern California for their hospitality during her talk in March 2017 and the AWM Research Symposium at UCLA in April 2017, where this work started.

\section{REFERENCES}

[1] Sami Assaf, Nonsymmetric Macdonald polynomials and a refinement of Kostka-Foulkes polynomials, to appear in Trans. Amer. Math. Soc.

[2] —_ Combinatorial models for Schubert polynomials, 2017, https://arxiv.org/abs/1703. 00088.

[3] _ Weak dual equivalence for polynomials, 2017, https://arxiv.org/abs/1702.04051.

[4] Sami Assaf and Dominic Searles, Schubert polynomials, slide polynomials, Stanley symmetric functions and quasi-Yamanouchi pipe dreams, Adv. in Math. 306 (2017), 89-122.

[5] Nantel Bergeron and Sara Billey, RC-graphs and Schubert polynomials, Experiment. Math. 2 (1993), no. 4, 257-269.

[6] I. N. Bernstein, I. M. Gel'fand, and S. I. Gel'fand, Schubert cells, and the cohomology of the spaces $G / P$, Uspehi Mat. Nauk 28 (1973), no. 3, 1-26.

[7] Sara Billey, William Jockusch, and Richard P. Stanley, Some combinatorial properties of Schubert polynomials, J. Algebraic Combin. 2 (1993), no. 4, 345-374.

[8] Daniel Bump and Anne Schilling, Crystal bases. representations and combinatorics, World Scientific Publishing Co. Pte. Ltd., Hackensack, NJ, 2017.

[9] Michel Demazure, Une nouvelle formule des caractères, Bull. Sci. Math. (2) 98 (1974), no. 3, 163-172.

[10] Paul Edelman and Curtis Greene, Balanced tableaux, Adv. in Math. 63 (1987), no. 1, 42-99.

[11] Jin Hong and Seok-Jin Kang, Introduction to quantum groups and crystal bases, Graduate Studies in Mathematics, vol. 42, American Mathematical Society, Providence, RI, 2002.

[12] Masaki Kashiwara, The crystal base and Littelmann's refined Demazure character formula, Duke Math. J. 71 (1993), no. 3, 839-858.

[13] Masaki Kashiwara and Toshiki Nakashima, Crystal graphs for representations of the q-analogue of classical Lie algebras, J. Algebra 165 (1994), no. 2, 295-345.

[14] Axel Kohnert, Weintrauben, Polynome, Tableaux, Bayreuth. Math. Schr. (1991), no. 38, 1-97, Dissertation, Universität Bayreuth, Bayreuth, 1990.

[15] Alain Lascoux and Marcel-Paul Schützenberger, Polynômes de Schubert, C. R. Acad. Sci. Paris Sér. I Math. 294 (1982), no. 13, 447-450.

[16] Schubert polynomials and the Littlewood-Richardson rule, Lett. Math. Phys. 10 (1985), no. $2-3,111-124$

[17] Keys 8 standard bases, in Invariant theory and tableaux (Minneapolis, MN, 1988), IMA Vol. Math. Appl., vol. 19, Springer, New York, 1990, pp. 125-144.

[18] Cristian Lenart, A unified approach to combinatorial formulas for Schubert polynomials, J. Algebraic Combin. 20 (2004), no. 3, 263-299.

[19] Peter Littelmann, Crystal graphs and Young tableaux, J. Algebra 175 (1995), no. 1, 65-87.

[20] I. G. Macdonald, Notes on Schubert polynomials, LACIM, Univ. Quebec a Montreal, Montreal, PQ, 1991.

[21] _ Schubert polynomials, in Surveys in combinatorics, 1991 (Guildford, 1991), London Math. Soc. Lecture Note Ser., vol. 166, Cambridge Univ. Press, Cambridge, 1991, pp. 73-99.

[22] Sarah Mason, An explicit construction of type A Demazure atoms, J. Algebraic Combin. 29 (2009), no. 3, 295-313.

[23] Cara Monical, Set-valued skyline fillings, Sém. Lothar. Combin. 78B (2017), Art. 35 (12 pages).

[24] Jennifer Morse and Anne Schilling, Crystal approach to affine Schubert calculus, Int. Math. Res. Not. (2016), no. 8, 2239-2294.

[25] Victor Reiner and Mark Shimozono, Key polynomials and a flagged Littlewood-Richardson rule, J. Combin. Theory Ser. A 70 (1995), no. 1, 107-143.

[26] , Plactification, J. Algebraic Combin. 4 (1995), no. 4, 331-351. 
[27] Richard P. Stanley, On the number of reduced decompositions of elements of Coxeter groups, European J. Combin. 5 (1984), no. 4, 359-372.

[28] John R. Stembridge, A local characterization of simply-laced crystals, Trans. Amer. Math. Soc. 355 (2003), no. 12, 4807-4823.

SAmi AssAf, Department of Mathematics, University of Southern California, 3620 S. Vermont Ave. Los Angeles, CA 90089-2532, U.S.A.

E-mail : shassaf@usc.edu

Anne Schilling, Department of Mathematics, UC Davis, One Shields Ave., Davis, CA 956168633, U.S.A.

E-mail : anne@math.ucdavis.edu 